\title{
DECOMPOSITIONS OF DUAL DISCRIMINATOR VARIETIES
}

\author{
R.J. Bignall
}

\begin{abstract}
A necessary and sufficient condition for a dual discriminator variety to be the product of a discriminator variety and a dual discriminator variety is given, along with an example of such a variety.
\end{abstract}

\section{INTRODUCTION}

The product $V_{1} \times V_{2}$ of two varieties $V_{1}$ and $V_{2}$ of the same type is the class of all algebras which are isomorphic to the direct product of a member of $V_{1}$ and a member of $V_{2} . V_{1}$ and $V_{2}$ are said to be independent if there is a term $s(x, y)$ such that $V_{1} \vDash s(x, y) \approx x$ and $V_{2} \vDash s(x, y) \approx y$. When $V_{1}$ and $V_{2}$ are independent their product is itself a variety. The product of two such independent varieties is denoted by $V_{1} \otimes V_{2}$. For details see Grátzer, Lakser and Plonka [3].

The ternary discriminator on a set $X$ is the function $t: X \rightarrow X$ given by

$$
t(a, b, c)= \begin{cases}a & \text { if } a \neq b \\ c, & \text { otherwise }\end{cases}
$$

The dual discriminator is the function $d: X \rightarrow X$ given by

$$
d(a, b, c)= \begin{cases}a & \text { if } a=b \\ c, & \text { otherwise }\end{cases}
$$

A variety $V$ is a discriminator variety (respectively, dual discriminator variety) if there is a term $w(x, y, z)$ such that $w^{\mathbf{A}}$ is the discriminator (respectively, dual discriminator) on $A$ for every subdirectly irreducible $\mathbf{A} \in V$. For more details see e.g. Burris and Sankappanavar, [1, Chapter IV], and Fried and Pixley [2].

Received 6 November 1990

Copyright Clearance Centre, Inc. Serial-fee code: 0004-9729/91 \$A2.00+0.00. 


\section{Separators}

Definition 2.1: A term $s(x, y, z)$ will be called a separator for a variety $V$ if it satisfies the following conditions.

(i) $s(x, s(x, y, z), z)$ induces the dual discriminator on every subdirectly irreducible member of $V$;

(ii) $V \models s(x, x, y) \approx s(y, x, x)$; and

(iii) $V \vDash s(x, s(x, s(x, y, z), z), z) \approx s(x, y, z)$.

One easily checks that a discriminator term for a discriminator variety $V$ is a separator for $V$. Likewise, a dual discriminator term is a separator for a dual discriminator variety.

Let $V$ denote a variety with a separator $s(x, y, z)$. Let $S$ be the class of subdirectly irreducible members of $V$. We will write $p(x, y, z)$ for the term $s(x, s(x, y, z), z)$.

LEмma 2.2. The following are identities of $V$.

(i) $p(x, y, y) \approx p(y, x, y) \approx p(y, y, x)$. Thus $p^{\mathbf{A}}$ is a majority polynomial on every $\mathbf{A} \in V$.

(ii) $s(x, y, x) \approx x \approx s(x, x, x)$.

(iii) $p(u, v, s(x, y, z)) \approx s(p(u, v, x), p(u, v, y), p(u, v, z))$.

Proof: (i) is an immediate consequence of the fact that $p(x, y, z)$ is a dual discriminator term. Part (ii) follows from 2.1 (iii). From the definition of the dual discriminator, and the fact that $s(u, u, u) \approx u$, it is easy to see that (iii) must hold on any subdirectly irreducible member of $V$. Thus (iii) must hold for all members of $V$.

Lemma 2.3. Let $\mathrm{A} \in S$ and let $\{a, b\} \subseteq A$ be such that $a \neq b$. Then one of the following two cases must hold for any $x, y \in\{a, b\}$.

CASE 1. $s^{\mathbf{A}}(x, y, y)=s^{\mathbf{A}}(y, y, x)=x$.

CASE 2. $s^{\mathbf{A}}(x, y, y)=s^{\mathbf{A}}(y, y, x)=y$.

Proof: Since $p^{\mathbf{A}}(x, y, z) \in\{x, z\}$ for all $x, y, z \in A$, we have, by 2.1 (iii), that $s^{\mathbf{A}}(x, y, z) \in\{x, z\}$ for any $x, y, z \in A$. In particular $\{a, b\}$ is closed under $s^{\mathbf{A}}$. Suppose that $s^{\mathbf{A}}(b, b, a)=s^{\mathbf{A}}(b, a, a)$. Without any loss of generality, we may assume that $s^{\mathbf{A}}(b, b, a)=a$. Then $b=p^{\mathbf{A}}(b, b, a)=s^{\mathbf{A}}\left(b, s^{\mathbf{A}}(b, b, a), a\right)=s^{\mathbf{A}}(b, a, a)=a$, which contradicts $a \neq b$. Hence $s^{\mathbf{A}}(b, b, a) \neq s^{A}(b, a, a)$, so we have either

$$
\begin{aligned}
& s^{\mathbf{A}}(a, b, b)=s^{\mathbf{A}}(b, b, a)=a, \text { and } \\
& s^{\mathbf{A}}(a, a, b)=s^{\mathbf{A}}(b, a, a)=b
\end{aligned}
$$

or

$$
\begin{aligned}
& s^{\mathbf{A}}(a, b, b)=s^{\mathbf{A}}(b, b, a)=b, \text { and } \\
& s^{\mathbf{A}}(a, a, b)=s^{\mathbf{A}}(b, a, a)=a .
\end{aligned}
$$


The result now follows, because $\boldsymbol{s}^{\mathbf{A}}$ is idempotent.

Lemma 2.4. Let $\mathbf{A} \in S$. Then $\boldsymbol{s}^{\mathbf{A}}$ is either the discriminator or the dual discriminator on $A$.

Proof: Let $a, u \in A$. Choose $b$ such that $a \neq b$. There are two cases to consider.

CASE 1. $s^{\mathbf{A}}(x, y, y)=s^{\mathbf{A}}(y, y, x)=x$ for all $x, y \in\{a, b\}$. Without loss of generality, we may suppose that $u \neq a$. If $s^{\mathbf{A}}(a, a, u)=a$, then $a=p^{\mathbf{A}}\left(b, a, s^{\mathbf{A}}(a, a, u)\right)=$ $s^{\mathbf{A}}\left(p^{\mathbf{A}}(b, a, a), p^{\mathbf{A}}(b, a, a), p^{\mathbf{A}}(b, a, u)\right)=s^{\mathbf{A}}(b, a, u)$.

But $\boldsymbol{s}^{\mathbf{A}}(b, a, u)$ must be either $b$ or $u$. This contradiction shows that case 1 of Lemma 2.3 holds for every two element subset of $A$. But then $s^{A}(x, y, y) \approx$ $s^{\mathbf{A}}(y, y, x) \approx x$ are identities of $\mathbf{A}$. Therefore, for any $u, v, w \in A$

$$
s^{\mathbf{A}}(u, v, w)=s^{\mathbf{A}}\left(u, p^{\mathbf{A}}(u, v, w), w\right)= \begin{cases}s^{\mathbf{A}}(u, u, w)=w & \text { if } v=u \\ s^{\mathbf{A}}(u, w, w)=u & \text { if } v \neq u\end{cases}
$$

Thus $\boldsymbol{s}^{\mathbf{A}}$ is the discriminator on $A$.

CASE 2. It is clear that if case 2 of Lemma 2.3 holds for $\{a, b\}$ then case 1 can not occur for any two element subset of $A$. Hence $\boldsymbol{s}^{\mathbf{A}}(x, y, y) \approx y$ and $s^{\mathbf{A}}(y, y, x) \approx y$ are identities of $\mathrm{A}$, and so for any $u, v, w \in A$

$$
\boldsymbol{s}^{\mathbf{A}}(u, v, w)=s^{\mathbf{A}}\left(u, p^{\mathbf{A}}(u, v, w), w\right)= \begin{cases}u & \text { if } v=u \\ v & \text { if } v \neq u\end{cases}
$$

Thus $\boldsymbol{s}^{\mathbf{A}}$ is the dual discriminator on $A$ in this case.

TheOREM 2.5. Let $V$ be a variety with a separator $s(x, y, z)$. Then there are subvarieties $V_{1}$ and $V_{2}$ of $V$ such that $s(x, y, z)$ induces the discriminator on every subdirectly irreducible member of $V_{1}$, and the dual discriminator on every subdirectly irreducible member of $V_{2}$. Furthermore, $V=V_{1} \otimes V_{2}$.

Proof: Let $S$ be the class of subdirectly irreducible members of $V$. We let $S_{1}=\left\{\mathrm{A} \in S: s^{\mathrm{A}}\right.$ is the discriminator on $\left.\mathrm{A}\right\}$, and $S_{2}=\left\{\mathrm{A} \in S: s^{\mathrm{A}}\right.$ is the dual discriminator on $\mathbf{A}\}$. Let $V_{1}$ and $V_{2}$ be the varieties generated by $V_{1}$ and $V_{2}$ respectively. In view of Lemma $2.4, V$ is the join of $V_{1}$ and $V_{2}$. Let $r(x, y)=s(x, y, y)$. Then $V_{1} \vDash r(x, y) \approx x$ and $V_{2} \vDash r(x, y) \approx y$. Since $V$ is a dual discriminator variety, it is congruence distributive, and the result now follows from Theorem 2 of [3].

The converse of Theorem 2.5 also holds.

THEOREM 2.6. Let $V$ be a variety containing subvarieties such that $V_{1}$ is a discriminator variety and $V_{2}$ is a dual discriminator variety, and $V=V_{1} \otimes V_{2}$. Then 
there is a term $s(x, y, z)$ of $V$ which induces the discriminator on the subdirectly irreducible members of $V_{1}$, and the dual discriminator on the subdirectly irreducible members of $V_{2}$. Such a term is a separator of $V$.

Proof: Since $V_{1}$ and $V_{2}$ are independent varieties, there is a term $r(x, y)$ such that $V_{1} \models r(x, y) \approx x$ and $V_{2} \vDash r(x, y) \approx y$. Let $t_{1}(x, y, z)$ be a discriminator term for $V_{1}$, and $d_{2}(x, y, z)$ be a dual discriminator term for $V_{2}$. Take $s(x, y, z)$ to be the term $r\left(t_{1}(x, y, z), d_{2}(x, y, z)\right)$. It is a straightforward matter to check that $s(x, y, z)$ has the required properties.

Since every discriminator variety is also a dual discriminator variety, we have

COROLlary 2.7. Two discriminator varieties of the same type are independent if and only if there is a term which is a discriminator term for one, and a dual discriminator term for the other.

\section{An Example}

Let $V$ be the variety of type $\langle 3\rangle$, with an operation symbol $s$ satisfying the identities:

$$
\begin{aligned}
& \text { (i) } s(x, y, y) \approx s(y, y, x) \\
& \text { (ii) } s(x, p(x, y, z), z) \approx s(x, y, z) \\
& \text { (iii) } s(x, y, z) \approx s(z, y, x) \\
& \text { (iv) } p(x, y, y) \approx p(y, x, y) \approx p(y, y, x) \approx y \\
& \text { (v) } p(z, p(x, y, z), p(x, y, w)) \approx p(x, y, z) \\
& \text { (vi) } p(u, v, s(x, y, z)) \approx p(u, v, s(u, v, x), s(u, v, y), s(u, v, z))
\end{aligned}
$$

where, as before, $p(x, y, z)$ is the term $s(x, s(x, y, z), z)$.

By [2, Theorem 3.2], $V$ is a dual discriminator variety, and by Theorem $2.5 \mathrm{~V}$ is the product of varieties $W$ and $U$, where $W$ is the quasi-primal variety whose only subdirectly irreducible member (up to isomorphism) is the two element set with the ternary discriminator as its operation, while $U$ is similarly generated by the the algebra with two elements and the dual discriminator as its operation. (Identity (iii) implies that the subdirectly irreducible members of $V$ have just two elements.)

$U$ is the variety of median algebras. These algebras were first studied by $M$. Sholander. They can be regarded as lower semilattices in which the supremum of any three elements exists if and only if they are pairwise bounded above. In addition, the infimum distributes over existing suprema. For details, see Sholander [4], [5] and in particular [5, Section 3].

\section{REFERENCES}

[1] S. Burris and H. P. Sankappanavar, A Course in Universal Algebra (Springer-Verlag, Berlin, Heidelberg, New York, 1980). 
[2] E. Fried and A. Pixley, 'The dual discriminator function in universal algebra', Acta Sci. Math. 41 (1979), 83-100.

[3] G. Grätzer, H. Lakser and J. Plonka, 'Joins and direct products of equational classes', Canad. Math. Bull. 12 (1976), 741-747.

[4] M. Sholander, 'Medians and betweenness', Proc. Amer. Math. Soc. 5 (1954), 801-807.

[5] M. Sholander, 'Medians, lattices and trees', Proc. Amer. Math. Soc 5 (1954), 808-812.

School of Applied Science

Monash University College Gippsland

Switchback Road

Churchill Vic 3842

Australia 